\title{
Seleção a campo de rizóbios nativos para cornichão
}

\author{
Field selection of native rhizobio for bird's-foot trefoil
}

\section{Marcos Roberto Dobler Stroschein ${ }^{\mathrm{I}}$ Marcelo Osório Wallau ${ }^{\mathrm{II}}$ Enilson Luiz Saccol de Sá ${ }^{I I I}$ Andréia Binz ${ }^{\mathrm{II}}$ Miguel Dall'Agnol ${ }^{\mathrm{IV}}$}

\section{- NOTA -}

\section{RESUMO}

O cornichão (Lotus corniculatus L.) tem sido apontado como uma das forrageiras mais promissoras para o uso no Rio Grande do Sul. No entanto, pouco se conhece a respeito da interação dessas plantas com os rizóbios nativos em condições de campo. Este estudo visou a avaliar a eficiência das interações entre rizóbios e plantas de Lotus corniculatus $c v$. 'São Gabriel' em condições de campo. $O$ experimento foi conduzido na Estação Experimental Agronômica da UFRGS com delineamento de blocos ao acaso com quatro repetições. Os tratamentos estudados foram: dois controles sem inoculação (um com a adição de $80 \mathrm{~kg} \mathrm{ha}^{-1}$ de nitrogênio na forma de uréia e outro sem a adição de nitrogênio); inoculação com rizóbios: estirpes SEMIA 816, recomendada no Brasil, U510, recomendada no Uruguai, U512, em estudo no Uruguai, e quatro isolados de rizóbios nativos (UFRGS Lc322, UFRGS Lc349, UFRGS Lc269 e Iso 7). O isolado UFRGS Lc322 e a estirpe U510 são mais eficientes do que a estirpe SEMIA 816 , recomendada para cornichão no país. Além disso, a produção de forragem das plantas inoculadas com o isolado UFRGS Lc322 e a estirpe U510 superou a obtida com a adição de $80 \mathrm{~kg} \mathrm{ha}^{-1}$ de nitrogênio.

Palavras-chave: Lotus corniculatus L., fixação biológica de nitrogênio, seleção de estirpes.

\section{ABSTRACT}

The bird's-foot trefoil (Lotus corniculatus L.) has been pointed as one of the most promising forage plants for use in Rio Grande do Sul. However, there is lack of information about the interaction of those plants with the native rhizobia in field conditions. This study aimed to evaluate the efficiency of interactions between rhizobia and plants of Lotus corniculatus $c v$. 'São Gabriel' in field conditions. The experiment was carried out in the Agronomic Experimental Station (EEA) of the Federal University of Rio Grande do Sul (UFRGS), using random block design with four replications. The treatments were: two controls without inoculation (one with the addition of urea corresponding to $80 \mathrm{~kg} \mathrm{ha}^{-1}$ of nitrogen and other without nitrogen addition); inoculation with rhizobia: strain SEMIA 816, recommended in Brazil, U150 recommended in Uruguay and U512, being studied in Uruguay, and four rhizobia isolates (UFRGS Lc322, UFRGS Lc349, UFRGS Lc269 e Iso 7). The rhizobia isolate Lc322 and the strain U510 are more efficient than the strain SEMIA 816, which is recommended for bird's-foot trefoil in the country. Furthermore, the forage production of plants inoculated with the isolate Lc322 and the strain U510 surpassed that, with the addition of $80 \mathrm{~kg} \mathrm{ha}^{-1}$ of nitrogen.

Key words: Lotus corniculatus L., biologic nitrogen fixation, strain selection.

Algumas espécies do gênero Lotus vêm sendo cultivadas no Rio Grande do Sul pelo seu potencial forrageiro, apresentando diversas vantagens que as tornam bem sucedidas, como melhor adaptabilidade a solos ácidos e crescimento em baixa disponibilidade de fósforo, fatores determinantes para sua razoável adaptação às condições ecológicas de pastagens naturais do sul do Brasil e norte do Uruguai e terras agricultáveis do nordeste do Uruguai e Argentina (BARAIBAR et al., 1999). Além disso, o seu

'Programa de Pós-graduação em Ciência do Solo, Universidade Federal do Rio Grande do Sul (UFRGS), 91540-000, Porto Alegre, RS, Brasil. E-mail: marcosstroschein@yahoo.com.br. Autor para correspondência.

"Curso de Agronomia, UFRGS, Porto Alegre, RS, Brasil.

"I'Departamento de Solos, UFRGS, Porto Alegre, RS, Brasil.

${ }^{\text {IV }}$ Departamento de Plantas Forrageiras, UFRGS, Porto Alegre, RS, Brasil. 
elevado teor de taninos beneficia a utilização com pastejo. Dentre as espécies de Lotus, o cornichão ( $\boldsymbol{L}$. corniculatus) tem sido apontado como uma das forrageiras mais promissoras para o uso no melhoramento de campos nativos no Rio Grande do Sul.

A interação entre rizóbios e plantas leguminosas apresenta grande potencial na sustentabilidade da produção dos sistemas agropecuários, devido às significativas entradas de nitrogênio no sistema solo-planta-animal, reduzindo a necessidade de fertilizantes nitrogenados e aumentando a disponibilidade de proteína para os animais. A espécie $\boldsymbol{L}$. corniculatus normalmente estabelece associações efetivas com Mesorhizobium sp., tendo sido observado grande variabilidade entre os isolados com relação à resposta da fixação biológica de nitrogênio (BARAIBAR et al., 1999), tornando necessário selecionar as bactérias mais eficientes. Atualmente, no país, existem poucas estirpes de rizóbio recomendadas para $L$. corniculatus e a maioria destas foram selecionadas na década de 70 e 80 (SCHEFFERBASSO et al., 2001). Nesse contexto, o objetivo deste estudo foi avaliar interações eficientes entre rizóbio e Lotus corniculatus cv. 'São Gabriel' em condições ambientais de campo.

O experimento foi conduzido na Estação Experimental Agronômica da UFRGS, em Argissolo vermelho, e conduzido durante o período de maio de 2007 a novembro de 2008 . Foi realizada a análise química do solo e a sua fertilidade foi corrigida com base nas recomendações de adubação e calagem para os estados do Rio Grande do Sul e Santa Catarina (ROLAS, 2004). A semeadura foi realizada em maio de 2007 e a inoculação dos rizóbios feita com auxílio de um aspersor manual 15 dias após a semeadura, para que não ocorresse favorecimento dos rizóbios inoculados, e, em cada parcela, foram adicionados $500 \mathrm{~mL}$ de um caldo de meio Levedura Manitol Agar contendo um número $1 \times 10^{8}$ células $\mathrm{mL}^{-1}$ de bactérias. Os tratamentos foram: dois controles sem inoculação (um com a adição de $80 \mathrm{~kg} \mathrm{ha}^{-1}$ de nitrogênio na forma de uréia, aplicados na semeadura e após cada corte, e outro sem a adição de nitrogênio); inoculação com rizóbios: estirpe SEMIA 816, recomendada no Brasil, U510, recomendada no Uruguai e U512, em estudo no Uruguai, e quatro isolados (UFRGS Lc322, UFRGS Lc349, UFRGS Lc269 e Iso. 7). O delineamento experimental foi o de blocos ao acaso com quatro repetições e as parcelas eram de $3 \mathrm{~m}^{2}$, com área útil de $1 \mathrm{~m}^{2}$.

Aos 90 dias após a semeadura, uma planta por parcela foi coletada e seu número de nódulos determinado. Não foi determinada a massa seca dos nódulos para que não ocorresse a destruição da amostra para posterior ensaio de competição (dados não mostrados). Para a quantificação dos valores de matéria seca, foram realizados dois cortes em cada parcela a $10 \mathrm{~cm}$ do solo, quando as plantas atingiram $5 \%$ da floração, com auxilio de um quadro amostral de $0,5 \mathrm{~m}^{2}$. Dessa amostra composta, foi retirada uma subamostra e seu peso fresco determinado. Essa mistura de plantas foi submetida à separação botânica entre as plantas de cornichão e inços e o material foi seco a $60^{\circ} \mathrm{C}$ até atingir um peso constante. O período dos cortes foi compreendido entre os meses de dezembro de 2007, fevereiro de 2008 e maio de 2008. Além desse parâmetro, também foram avaliados o teor de nitrogênio na parte aérea (TEDESCO et al., 1995). Os valores de nitrogênio obtidos foram utilizados para calcular o índice de eficiência relativa na fixação biológica de nitrogênio, conforme proposto por BROCKWELL et al. (1966) e definido através da

seguinte expressão: $\operatorname{Ef}(\%)=\frac{\left(N_{\text {iso }}-N_{T-N}\right)}{\left(N_{T+N} 1 N_{T-N}\right)} \times \mathbf{1 0 0}$

Sendo: $\operatorname{Ef}(\%)=$ eficiência relativa na fixação biológica de nitrogênio; $\mathrm{N}_{\text {iso }}=$ nitrogênio total do tratamento inoculado; $\mathrm{N}_{\mathrm{T}+\mathrm{N}}=$ nitrogênio total do controle com adição de nitrogênio; $\mathrm{N}_{\mathrm{T}-\mathrm{N}}=$ nitrogênio total do tratamento controle sem adição de nitrogênio.

Não foram observadas diferenças significativas no número de nódulos, no entanto, nas plantas inoculadas com a estirpe U510 e o isolado UFRGS Lc322, foram encontrados 135 e 132 nódulos, respectivamente, apresentando cerca de $50 \%$ mais nódulos do que as plantas controle, mas, devido ao elevado coeficiente de variação, estes valores não diferiram (Tabela 1). Estes resultados foram superiores aos encontrados por FRIZZO (2007) e inferiores aos de FONTOURA (2007) em casa de vegetação.

As plantas inoculadas com o isolado UFRGS Lc322 e com a estirpe U510 apresentaram os maiores valores de nitrogênio total na parte aérea, tendo acumulado, respectivamente, 43 e $34 \mathrm{~kg} \mathrm{ha}^{-1}$ a mais que o controle com adição de $80 \mathrm{~kg}$ de $\mathrm{N} \mathrm{ha}^{-1}$. Os resultados do tratamento inoculado com a estirpe SEMIA $816 \mathrm{e}$ Iso7 não diferiram do controle com nitrogênio. As plantas inoculadas com a estirpe U512 e com os isolados UFRGS Lc349 e UFRGS Lc269 não diferiram das plantas sem a adição nitrogênio. Os índices de eficiência relativa calculados para os tratamentos inoculados variaram de 225 a 4\% (Tabela 1). O isolado UFRGS Lc322 e a estirpe U510 apresentaram maior eficiência relativa, sendo superiores a $175 \%$. Os tratamentos inoculados com as estirpes SEMIA 816 e U512 não diferiram do tratamento controle sem nitrogênio. A maior produção de matéria seca foi obtida nas plantas inoculadas com 
Tabela 1 - Número de nódulos, nitrogênio total acumulado em dois cortes da parte aérea e eficiência relativa na fixação biológica de nitrogênio em plantas de Lotus corniculatus inoculadas com rizóbios. Corte realizado no mês de dezembro de 2007.

\begin{tabular}{|c|c|c|c|}
\hline Tratamentos & Nódulos planta ${ }^{-1}$ & $\begin{array}{l}\text { Nitrogênio Total } \\
\qquad \mathrm{kg} \mathrm{ha}^{-1}\end{array}$ & $\begin{array}{c}\text { ER } \\
\%\end{array}$ \\
\hline $\mathrm{T}+\mathrm{N}$ & $61 \mathrm{~ns}$ & $90 \mathrm{~b}^{*}$ & $100 \mathrm{~b}$ \\
\hline UFRGS Lc322 & 132 & $133 \mathrm{a}$ & $225 \mathrm{a}$ \\
\hline U 510 & 135 & $124 \mathrm{a}$ & $175 \mathrm{a}$ \\
\hline Iso.7 & 85 & $97 \mathrm{~b}$ & $80 \mathrm{~b}$ \\
\hline SEMIA 816 & 91 & $80 \mathrm{~b}$ & $45 \mathrm{c}$ \\
\hline U 512 & 99 & $70 \mathrm{c}$ & $8 \mathrm{c}$ \\
\hline UFRGS Lc269 & 88 & $57 \mathrm{c}$ & $8 \mathrm{c}$ \\
\hline UFRGS Lc349 & 109 & $64 \mathrm{c}$ & $4 \mathrm{c}$ \\
\hline $\mathrm{T}-\mathrm{N}$ & 68 & $78 \mathrm{c}$ & $0 \mathrm{c}$ \\
\hline $\mathrm{CV}(\%)$ & 78,3 & 13,4 & 18,7 \\
\hline
\end{tabular}

$\mathrm{T}+\mathrm{N}=$ Tratamento controle com adição de nitrogênio; $\mathrm{T}-\mathrm{N}=$ Tratamento controle sem adição de nitrogênio. ns: Não significativo a 5\% de probabilidade de erro.*Médias seguidas pela mesma letra na coluna não diferem significativamente pelo teste Scott-Knott 5\%.

o isolado UFRGS Lc322 e com a estirpe U510 (9,5 e $9,8 \mathrm{Mg} \mathrm{ha}^{-1}$, respectivamente), sendo superior aos tratamentos controle com e sem adição de nitrogênio e inoculado com a estirpe SEMIA 816. Os tratamentos inoculados com a estirpe SEMIA 816, recomendada para o cultivo de $\boldsymbol{L}$. corniculatus no Brasil, e com os isolados UFRGS Lc349 e estirpe U512 não diferiram do controle sem adição de nitrogênio (Figura 1).

\section{CONCLUSÕES}

a) O isolado de rizobio nativo UFRGS Lc322 e a estirpe U510 são mais eficientes, em condições de campo, do que a estirpe SEMIA 816.

b) A produção de forragem das plantas inoculadas com o isolado UFRGS Lc322 e a estirpe U510 foram maiores do que a obtida nas plantas que receberam adubação com ureia.

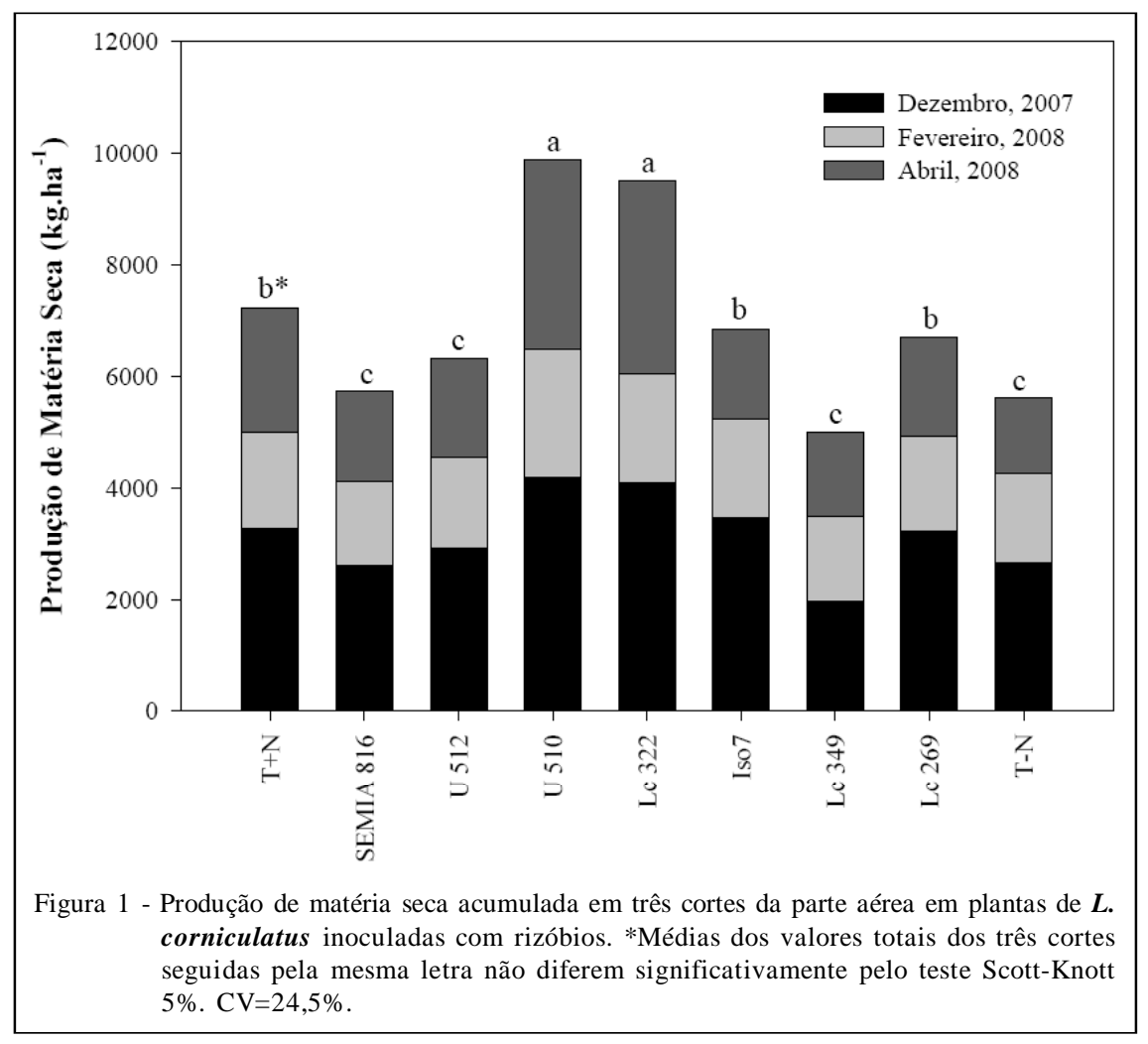

Ciência Rural, v.41, n.10, out, 2011. 


\section{REFERÊNCIAS}

BARAIBAR, A. et al. Symbiotic effectiveness and ecological characterization of indigenous Rhizobium loti populations in Uruguay. Pesquisa Agropecuária Brasileira, Brasília, v.34, n.6, p. 1011-1017, 1999. Disponível em: <www.scielo.br/ scielo.php ? script $=$ sci_arttext \& pid=S $0100-$ 204X1999000600012>. Acesso em: 11 jun. 2009. doi: 10.1590/S0100-204X1999000600012.

BROCKWELL, J. et al. Some symbiotic characteristics of rhizobia responsible for spontaneous, effective field nodulation of Lotus hispidus. Australian Journal of Experimental Agriculture and Animal Husbandry, Tamworth, v.6, p.365370, 1966. Disponível em: <www.publish.csiro.au/paper/ EA9660365.htm>. Acesso em: 11 jun. 2009. doi: 10.1071/ EA9660365.

FONTOURA, R.A. Seleção de rizóbios nativos, de solos do Rio Grande do Sul, para Lotus glaber e L. subbiflorus. 2007. 83f. Dissertação (Mestrado em Microbiologia Agrícola) - Curso de Pós-graduação em Microbiologia Agrícola, Universidade Federal de Santa Maria, RS.
FRIZZO, M.L. dos S. Seleção e caracterização de rizóbios nativos, de solos do Rio Grande do Sul, para Lotus corniculatus L. e Lotus uliginosus Schkuhr. 2007. 87. Dissertação (Mestrado em Ciência do Solo) - Curso de Pós-graduação em Ciência do Solo, Universidade Federal do Rio Grande do Sul, RS.

ROLAS. Manual de adubação e calagem para os estados do Rio Grande do Sul e Santa Catarina. 10.ed. Porto Alegre: Sociedade Brasileira de Ciência do Solo - Núcleo Regional Sul, 2004. 394p.

SCHEFFER-BASSO, S.M. et al. Nodulação e fixação biológica de nitrogênio de Adesmia latifolia e Lotus corniculatus em vasos de Leonard. Revista Brasileira de Zootecnia, Viçosa, v.30, n.3, p.687-693, 2001. Disponível em: <http:// w w w. s c i e lo.br/s c i e 1 o.ph p ? p id = S 1516 35982001000300012\&script=sci_arttext $>$. Acesso em: 11 jun. 2009. doi: 10.1590/S1516-35982001000300012.

TEDESCO, M.J. et al. Análise de solo, planta e outros materiais. 2.ed. Porto Alegre: UFRGS/DS, 1995. 443p. (Boletim Técnico, 5). 